\title{
Capacitively Loaded Loop-Based Antennas with Reconfigurable Radiation Patterns
}

\author{
Saber Dakhli, ${ }^{1}$ Hatem Rmili, ${ }^{2}$ Jean-marie Floc'h, ${ }^{3}$ Muntasir Sheikh, ${ }^{2}$ \\ Kourosh Mahdjoubi, ${ }^{1}$ Fethi Choubani, ${ }^{4}$ and Richard W. Ziolkowski ${ }^{2,5}$ \\ ${ }^{1}$ IETR, University of Rennes 1, Campus Beaulieu, Bât. 11D, No. 263, avenue Général Leclerc, CS 74205, 35042 Rennes Cedex, France \\ ${ }^{2}$ Electrical and Computer Engineering Department, King Abdulaziz University, P.O. Box 80204, Jeddah 21589, Saudi Arabia \\ ${ }^{3}$ IETR, INSA, 20 avenue Buttes de Coësmes, 35043 Rennes, France \\ ${ }^{4}$ Innov'Com Laboratory, SUPCOM, University of Carthage, Tunis, Tunisia \\ ${ }^{5}$ Department of Electrical and Computer Engineering, University of Arizona, Tucson, AZ, USA
}

Correspondence should be addressed to Hatem Rmili; hatem.rmili@yahoo.fr

Received 23 January 2015; Revised 31 March 2015; Accepted 31 March 2015

Academic Editor: Vincenzo Galdi

Copyright (c) 2015 Saber Dakhli et al. This is an open access article distributed under the Creative Commons Attribution License, which permits unrestricted use, distribution, and reproduction in any medium, provided the original work is properly cited.

\begin{abstract}
A class of metamaterial-inspired antennas having reconfigurable radiation patterns is proposed. They consist of a driven monopole antenna with one- and two-capacitively loaded loop (CLL), near field resonant parasitic elements. Two configurations are studied by considering the state of these CLL elements as being either open or closed configurations. Simulation results explain the design features and demonstrate that the structure can change its beam direction simply by controlling the switched states. Two prototypes with one- and two-CLL elements were fabricated and tested. The measured impedance mismatch and radiation pattern results are presented and compared to the corresponding simulated values.
\end{abstract}

\section{Introduction}

The interest in and demand for wireless and mobile platforms have grown dramatically in the past decade. Applications include, for example, communications, sensors, RFID tags, and health monitoring systems. The need to increase the functionality of the corresponding antenna systems within the same or smaller footprints in these systems has led to a host of investigations into nontraditional solutions.

One approach is the use of metamaterial-inspired antennas $[1,2]$. Metamaterials are artificial materials that can be designed to exhibit unusual electromagnetic properties, such as simultaneous negative permittivity and permeability [3]. These unusual properties have been shown to provide unconventional paths to novel and improved antennas with interesting and desirable performance characteristics $[2,4]$. Different classes of metamaterial-inspired electrically small antennas, which are efficient and matched intrinsically to the source, have been designed to have multifunctional properties, for example, multiband and circular polarization, using near field resonant parasitic (NFRP) elements [5]. A variety of these designs have included the introduction of capacitively loaded loop (CLL) and split ring resonator (SRR) elements as the NFRP elements [1, 2, 4-10], as artificial magnetic conductors (AMCs) to achieve hemispherical coverage [11], and as local resonators to achieve notched filters in UWB antennas [12-14].

A second approach is to introduce reconfigurable antennas $[15,16]$. A variety of components have been investigated to achieve reconfigurability. These include mechanically movable parts [16]; electronic switches including semiconductor devices [17], such as MEMS [18] and varactors [19]; and optically activated switches [20]. Numerous antenna performance characteristics have become tunable with reconfigurable elements.

In this paper, we introduce a class of metamaterialinspired antennas having reconfigurable radiation patterns. It is achieved by combining two previous directive configurations. In the work [21], a family of efficient metamaterialinspired antennas was studied that was composed of a 


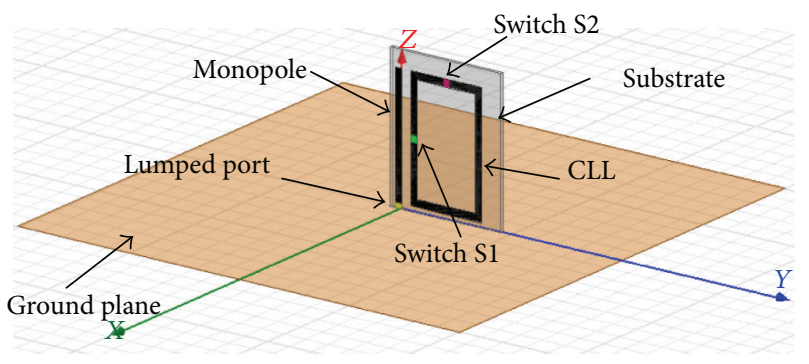

(a)

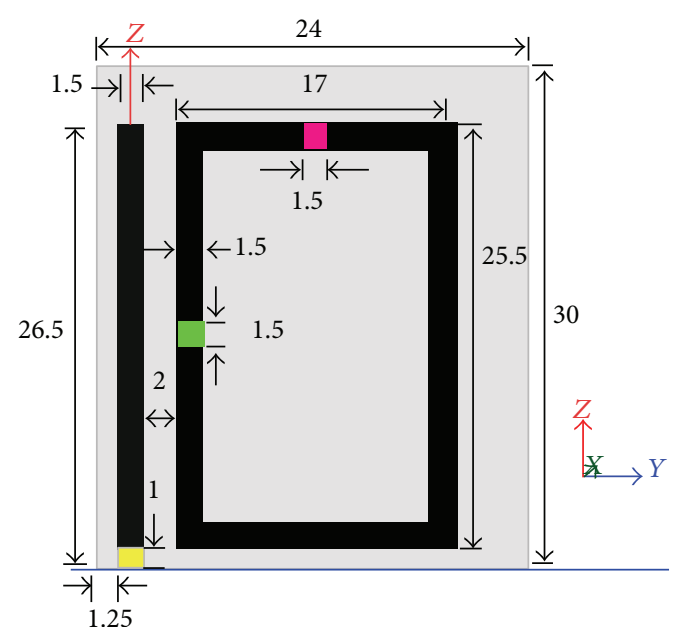

(b)

FIGURE 1: (a) Simulation model of the single-CLL, two-gap element-based reconfigurable antenna, and (b) geometry of the individual elements (dimensions in millimeters).

monopole antenna with a SRR NFRP element. Four configurations of the antenna were studied by changing the position of the gap in the SRR loop. It was shown that, among the four studied configurations, only two exhibit high directivities. In the work [22], it was demonstrated that the location of the gap of the CLL NFRP element could be used to change the main beam directions of a similar electrically small metamaterialinspired antenna. The structures presented here consist of a monopole antenna combined with one and two CLL-based NFRP elements that include two open-closed configurations, realized with switches, to achieve the reconfigurable patterns. It is demonstrated with simulation results that both of these structures change their main beam directions depending on the states of those switches. Prototypes of both antennas were fabricated and tested. The measured results confirm the predicted behaviors.

In comparison to a previous report of the same concept [23], the CLL elements employed here are much simpler than the SRR elements. As a result, the antennas reported here have less flexibility since they deal only with two switches rather than four. On the other hand, the CLL elements achieve much higher radiation efficiencies. In addition to simplifying the associated switching network, the emphasis here is simply to switch the directivity from one direction to the opposite one. Moreover, because they are assumed to have a (finite) ground plane, their intended applications are different. The antennas reported here, for instance, could be integrated into a variety of vehicles and other transportation systems. As a consequence of their geometry, the monopoles of our antenna systems are directly fed from a SMA connector rather than using an intermediate CPW feed. Furthermore, experimental results are provided to confirm the design concepts.

\section{Antenna with One CLL Element}

The single-CLL-based antenna was designed and simulated using the ANSYS-HFSS software. It was fabricated with a Rogers Duroid 5880 substrate with a thickness of $0.8 \mathrm{~mm}$ and relative permittivity $\varepsilon_{r}=2.2$, relative permeability $\mu_{r}=$ 1.0, and 0.0009 loss tangent. The antenna was excited in the simulation models with a lumped port having a $50 \Omega$ input impedance.

2.1. Antenna Design. The single-CLL NFRP antenna consists of a driven monopole of length $25.5 \mathrm{~mm}$ and width of $1.5 \mathrm{~mm}$ printed on the Rogers substrate (see Figure 1). The CLL element has a $25.5 \mathrm{~mm}$ length and $17 \mathrm{~mm}$ width. It includes two open gaps having $1.5 \times 1.5 \mathrm{~mm}^{2}$ dimensions as shown in Figure 1(a). The dimensions of the ground plane are $100 \times 100$ $\mathrm{mm}^{2}$.

The antenna system was designed to operate at $1.55 \mathrm{GHz}$. Two configurations were considered that depend on the state of switches S1 and S2 in Figure 1(a). Details of the design parameters are given in Figure 1(b). The states ON and OFF of each switch correspond to a closed CLL (short circuit for the CLL-gap) and an open CLL (open circuit for the CLL-gap), respectively.

2.2. Simulation Results. The simulated impedance mismatch, that is, the $\left|S_{11}\right|$ values, of the single NFRP element reconfigurable antenna is presented in Figure 2. As shown, when switch S1 is ON and S2 is OFF (State 1), the structure operates at $f_{R}=1.55 \mathrm{GHz}$. On the other hand, when the switch S2 is ON and S1 is OFF (State 2), the antenna resonance is at $f_{R}=1.72 \mathrm{GHz}$. The $-10 \mathrm{~dB}$ fractional bandwidth is $2 \%$ for State 1 and $0.6 \%$ for State 2.

Figure 3 illustrates the simulated surface current vectors of the two configurations at their resonance frequencies. We note from this figure that the current distributions depend on which switch is in its open position. In fact, this open switch location in the CLL element affects both the orientation and magnitude of the currents. These current distributions help explain the associated radiation pattern behaviors. 


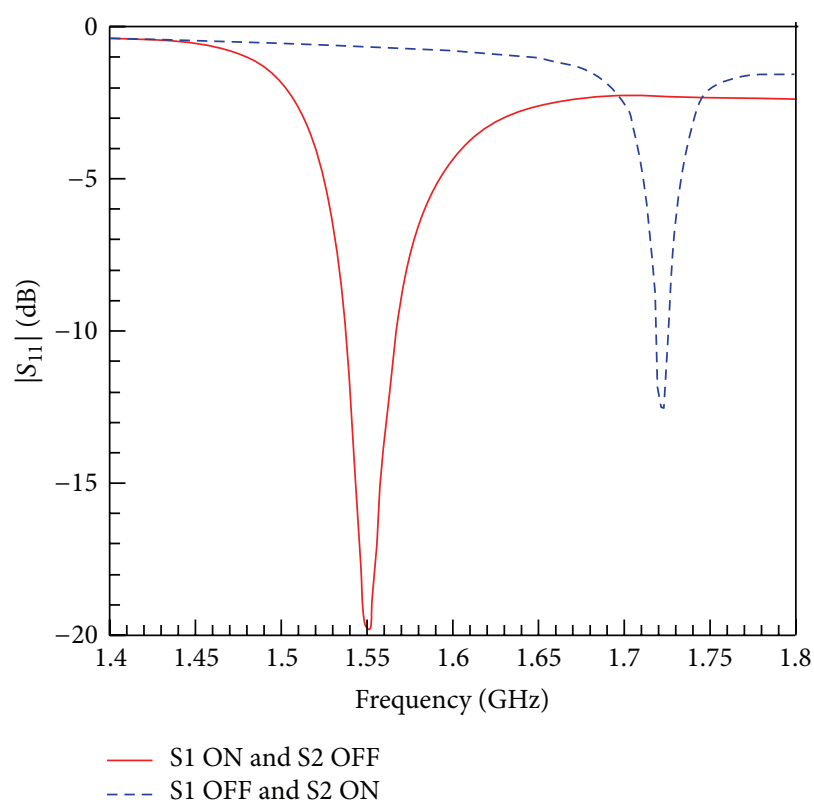

FIGURE 2: Simulated input impedance mismatch, $\left|S_{11}\right|$, as a function of the source frequency of the proposed single NFRP antenna.

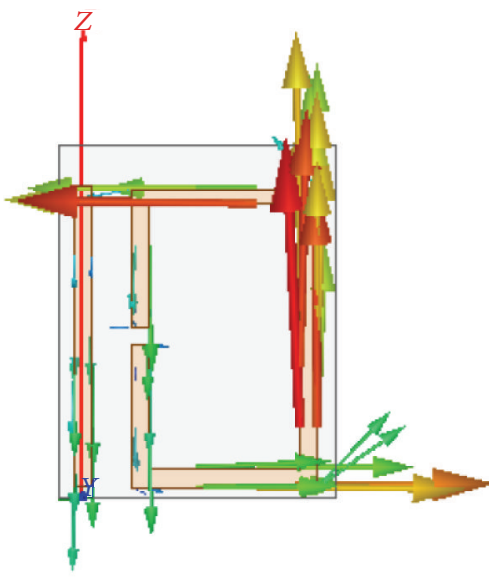

(a)

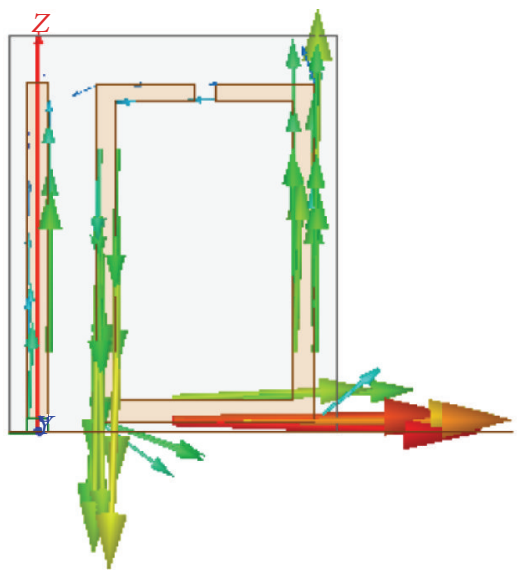

(b)

FIGURE 3: Simulated surface current for the single NFRP element reconfigurable antenna. (a) S1 ON and S2 OFF ( $\left.f_{R}=1.55 \mathrm{GHz}\right)$, and (b) S1 OFF and $\mathrm{S} 2 \mathrm{ON}\left(f_{R}=1.72 \mathrm{GHz}\right)$.

The corresponding simulated 3D radiation patterns of the antenna are given in Figure 4. These patterns correspond to the resonant frequencies $1.55 \mathrm{GHz}$ and $1.72 \mathrm{GHz}$, respectively. One observes in Figure 4(a) that the antenna radiates more towards the negative $Y$-direction, opposite to the CLL element, at the lower resonance frequency. In contrast, Figure 4(b) shows that the structure radiates primarily towards the positive $Y$-direction at the higher resonance frequency. These two pattern changes are correlated to the major changes in the currents along the vertical strips (the driven monopole and the sides of the CLL element parallel to the $Z$-axis) shown in Figure 3. The simulated maximum radiation efficiency for State 1 and State 2 is, respectively, $80 \%$ and $60 \%$, whereas the maximum realized gain is $1 \mathrm{~dB}$ and $-1.5 \mathrm{~dB}$, respectively.
Figure 5 gives the simulated 2D radiation patterns at both resonance frequencies in the (XOY) plane. When switch $\mathrm{S} 1$ is $\mathrm{ON}$ ( $\mathrm{S} 2$ is $\mathrm{OFF}$ ), the direction of the beam at the lower resonance frequency is toward the negative $Y$-direction. On the other hand, when the switch S2 is ON (S1 is OFF), the beam at the higher resonance frequency changes its direction toward the positive $Y$-direction.

2.3. Experimental Validation. A prototype of the single NFRP element antenna with the two open gaps was fabricated and characterized. Figure 6 presents a photo of the realized prototype. Both impedance mismatch and radiation patterns were measured.

The impedance mismatch, that is, the $\left|S_{11}\right|$ values, as a function of the source frequency was measured using a 

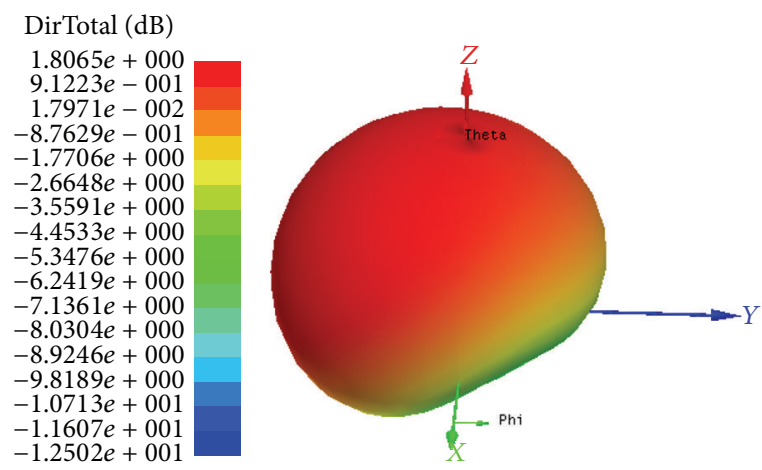

(a)
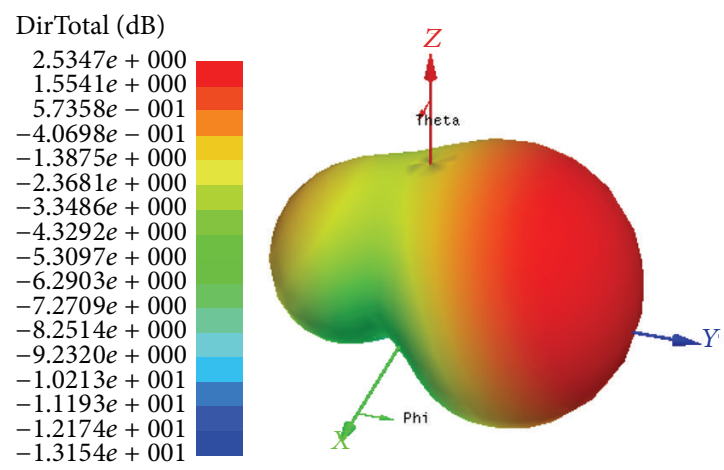

(b)

FIGURE 4: Simulated 3D radiation patterns for the single NFRP element reconfigurable antenna. (a) S1 ON and S2 OFF $\left(f_{R}=1.55 \mathrm{GHz}\right)$, and (b) S1 OFF and S2 ON $\left(f_{R}=1.72 \mathrm{GHz}\right)$.

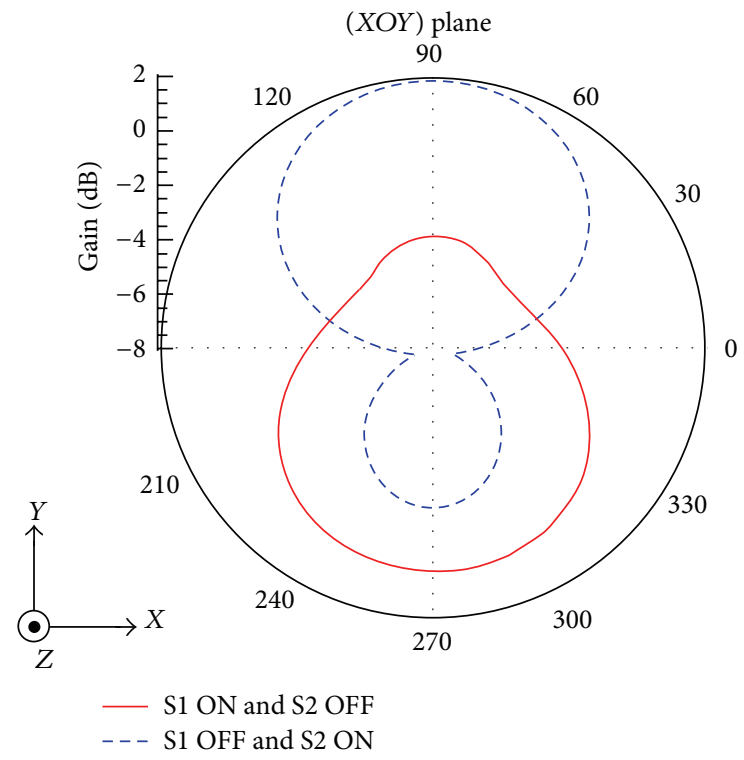

FIGURE 5: Simulated radiation patterns of the single NFRP element reconfigurable antenna in the $(X O Y)$ plane. State 1 (red) at $f_{R}=$ $1.55 \mathrm{GHz}$ and State 2 (blue) at $f_{R}=1.72 \mathrm{GHz}$.

network analyzer Agilent N5230A over the frequency range $1.5-2.5 \mathrm{GHz}$. Figure 7 presents the measured $\left|S_{11}\right|$ values. According to Figure 8, when switch $\mathrm{S} 1$ is $\mathrm{ON}$ (S2 is OFF), the structure operates at $1.59 \mathrm{GHz}$, whereas when the switch S2 is $\mathrm{ON}$ ( $\mathrm{S} 1$ is $\mathrm{OFF}$ ), the resonance is at $1.76 \mathrm{GHz}$. The measured results are in very good agreement with the simulation values. For example, the simulated and measured fractional bandwidths corresponding to State 1 are, respectively, $2.0 \%$ and $1.9 \%$. The measured $\left|S_{11}\right|$ value fell above $-10 \mathrm{~dB}$. The corresponding $-3 \mathrm{~dB}$ are $2.3 \%$ and $1.7 \%$ for State 2 .

Figure 8 presents the measured radiation patterns of the prototype antenna in the $(X O Y)$ plane at $f_{R}=1.59 \mathrm{GHz}$ and $f_{R}=1.76 \mathrm{GHz}$. These measurements were carried out in a "SATIMO Stargate32" anechoic chamber. As shown in Figure 8, when switch $\mathrm{S} 1$ is $\mathrm{ON}$ (S2 is OFF), the direction of the beam, as predicted, is towards the negative $Y$-direction at

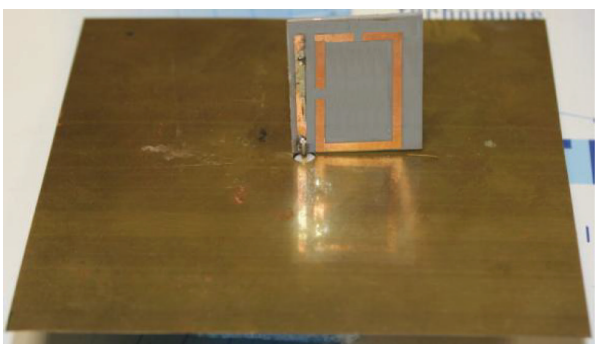

FIGURE 6: Photo of the realized prototype of the single NFRP element reconfigurable antenna.

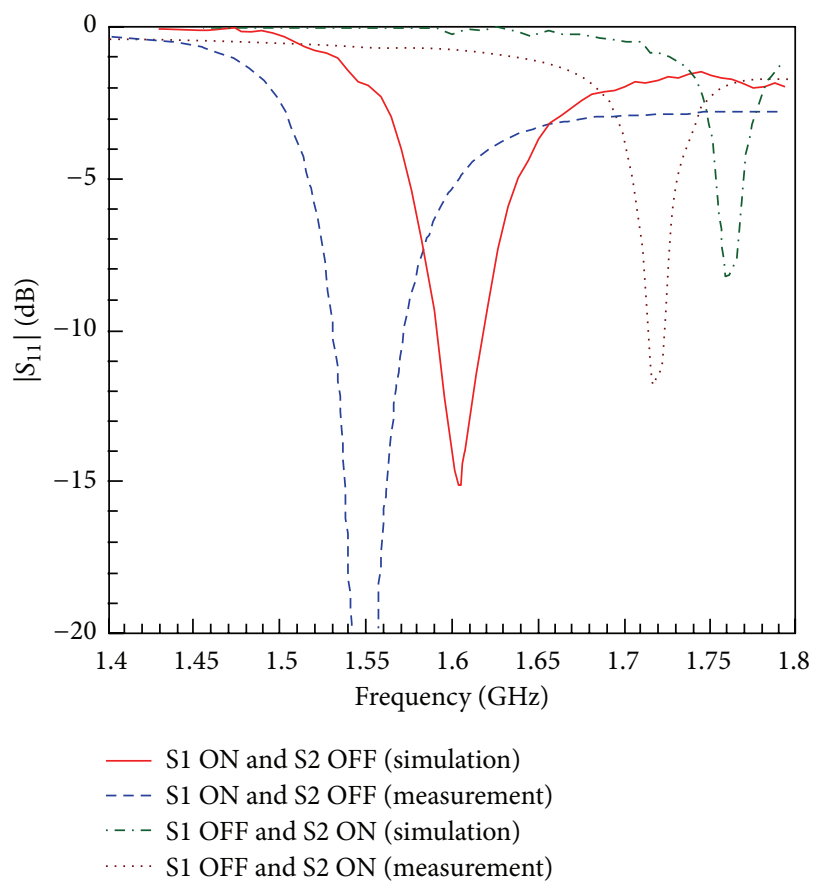

FIgURE 7: Measured and simulated impedance mismatch of the fabricated prototype of the single NFRP element reconfigurable antenna. 


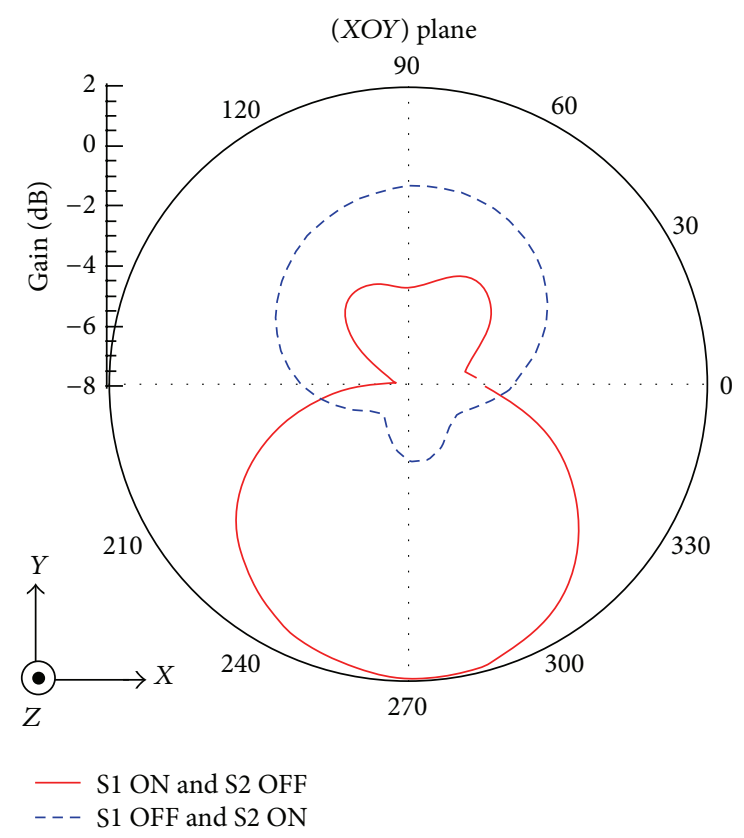

FIGURE 8: Measured 2D radiation patterns in the (XOY) plane of the prototype single NFRP element reconfigurable antenna. State 1 (red) at $f_{R}=1.59 \mathrm{GHz}$ and State 2 (blue) at $f_{R}=1.76 \mathrm{GHz}$.

$1.59 \mathrm{GHz}$. On the other hand, when the switch S2 is $\mathrm{ON}$ ( $\mathrm{S} 1$ is OFF), the beam changes its direction, as predicted, towards the positive $Y$-direction at $1.76 \mathrm{GHz}$.

The corresponding measured $3 \mathrm{D}$ radiation patterns of the prototype single NFRP element antenna are shown in Figure 9. Again, the agreement with the predicted patterns given in Figure 4 is good. The simulated maximum values of the radiation efficiency are $80 \%$ and $60 \%$ for States 1 and 2, respectively.

\section{Antenna with Two CLL Elements}

The two-CLL-based NFRP antenna was also designed and simulated using the ANSYS-HFSS software. It was fabricated and measured with the same experimental conditions as the one-CLL-based NFRP antenna presented in Section 2. Some design parameters were changed compared to the previous structure with $1 \mathrm{CLL}$ element in order to obtain lower operating frequencies.

3.1. Antenna Design. The antenna consists of a monopole of length $29 \mathrm{~mm}$ and width $2 \mathrm{~mm}$ coupled with two identical CLL elements of length $29 \mathrm{~mm}$ and the width $21 \mathrm{~mm}$. Each CLL element is loaded with a gap of dimensions $2 \times$ $2 \mathrm{~mm}^{2}$. The other design parameters remained. One switch is located in each CLL element (Figure 10(a)) in order to ensure the antenna reconfigurability. Details of the design of this reconfigurable structure are given in Figure 10.

As shown in Figure 11, the antenna with either S1 or S2 open operates at the same resonance frequency $f_{R}=$ $1.172 \mathrm{GHz}$. A second resonance frequency occurs outside the band of interest. This primary frequency corresponds to the resonance of only one capacitor-loaded NFRP element. We note that the two sates operate at the same frequency because the structure is perfectly symmetric, which explains the coincidence of the $\left|S_{11}\right|$ curves corresponding to both States 1 and 2, respectively. The $-10 \mathrm{~dB}$ fractional bandwidth for both States 1 and 2 is $2.6 \%$.

The simulated surface current vectors of the two configurations at the same resonance frequency $f_{R}=1.172 \mathrm{GHz}$ are illustrated in Figure 12. We note from this figure that the current distributions depend on which switch is activated, that is, which CLL element is resonant. In fact, when the switch is ON, the CLL element corresponds to a closed loop and does not resonate, whereas when it is open, it resonates. We also notice that the current distributions, as would be expected, are symmetric. This behavior confirms the previous discussion of the coincident $\left|S_{11}\right|$ results at the same frequency.

Figure 13 illustrates the $2 \mathrm{D}$ simulated radiation patterns for the proposed structure. When switch $\mathrm{S} 1$ is $\mathrm{ON}$ ( $\mathrm{S} 2$ is $\mathrm{OFF}$ ), the direction of the beam is toward the negative $Y$-direction, whereas when the switch S2 is $\mathrm{ON}$ ( $\mathrm{S} 1$ is $\mathrm{OFF}$ ), the beam changes its direction toward the positive $Y$-direction. A major advantage of this structure, in contrast to the single-CLL NFRP element antenna, is the fact that this reconfigurability of the pattern occurs at the same frequency. The maximum values of the simulated radiation efficiency and realized gain, corresponding to both States 1 and 2, are approximately $80 \%$ and $0 \mathrm{~dB}$, respectively.

3.2. Experimental Validation. A prototype of the two-CLL NFRP element antenna with each CLL element having one open gap was fabricated. The antenna was then characterized with only one of the gaps switched on. Figure 14 presents a photo of the realized prototype in the measurement chamber. The impedance mismatch, that is, the $\left|S_{11}\right|$ values, as a function of the source frequency was measured using an Agilent N5230A network analyzer over the frequency range $1-1.4 \mathrm{GHz}$.

Figure 15 gives the measured $\left|S_{11}\right|$ values as a function of the source frequency from 1 to $1.4 \mathrm{GHz}$. As shown in Figure 15, the structure with either S1 or S2 open operates at the same resonance frequency $f_{R}=1.180 \mathrm{GHz}$. The measured $-10 \mathrm{~dB}$ fractional bandwidth was $2.5 \%$ for State 1 , in very good agreement with the simulated value: $2.6 \%$. It is only $0.9 \%$ for State 2 , indicating that fabrication errors were introduced with opening and closing the CLL elements.

Figure 16 presents the measured radiation pattern in (XOY) plane of the studied antenna at the resonance frequencies. These measurements also were carried out in the anechoic chamber "SATIMO Stargate32." When switch S1 is $\mathrm{ON}$ (S2 is OFF), the direction of the beam is toward the negative $Y$-direction, whereas when the switch S2 is ON (S1 is $\mathrm{OFF}$ ), the beam changes its direction toward the positive $Y$-direction. These results are in excellent agreement with the simulated ones.

The corresponding measured $3 \mathrm{D}$ radiation patterns of the prototype two-NFRP element antenna are shown in Figure 17. We notice in Figure 17 that the reconfigurability 


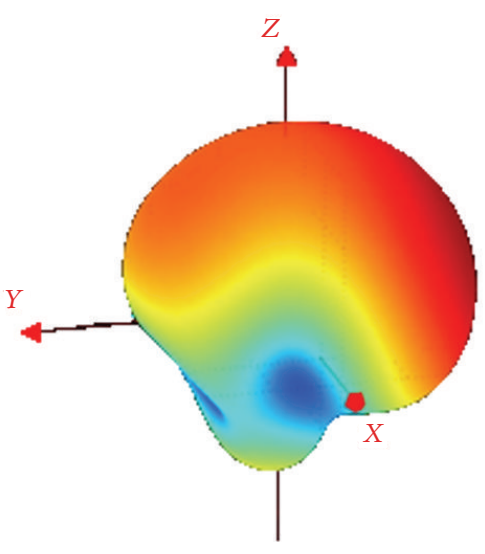

(a)

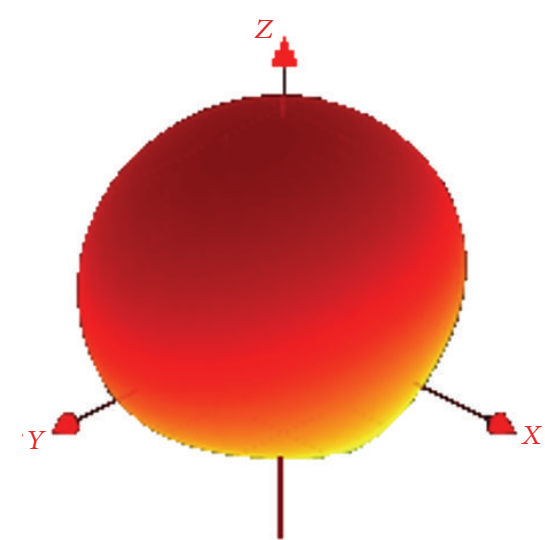

(b)

FIGURE 9: Measured 3D radiation patterns of the prototype single NFRP element reconfigurable antenna. (a) State 1 at $f_{R}=1.59 \mathrm{GHz}$ and (b) State 2 at $f_{R}=1.76 \mathrm{GHz}$.

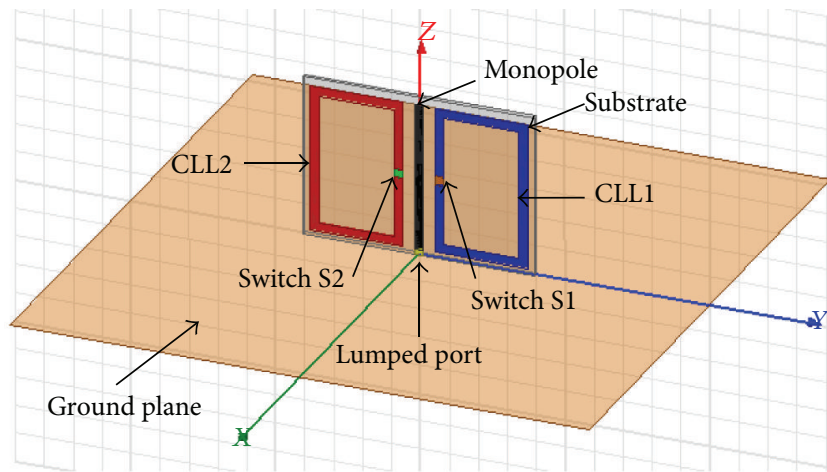

(a)

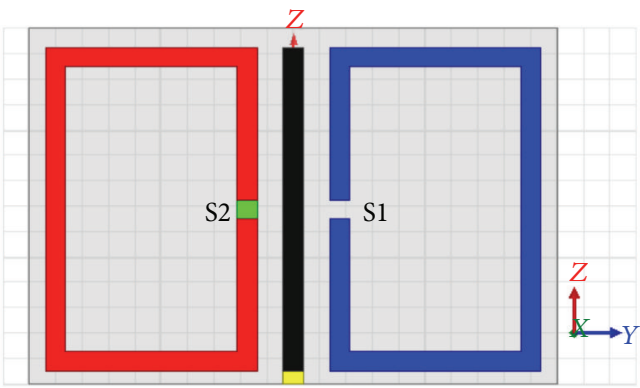

(b)

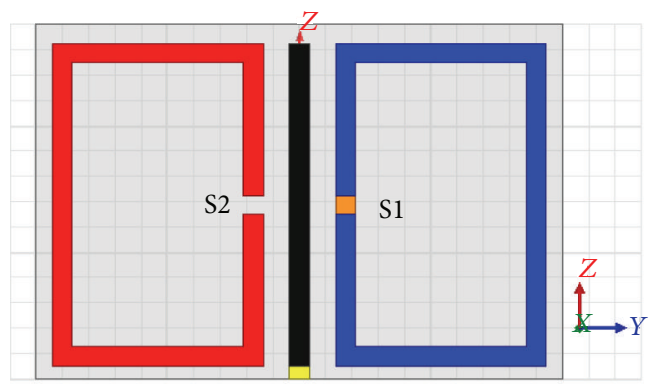

(c)

Figure 10: (a) Simulation model of the two-CLL element NFRP reconfigurable antenna, (b) State 1 (S1 is ON and S2 is OFF), and (c) State 2 ( $\mathrm{S} 2$ is $\mathrm{ON}$ and $\mathrm{S} 1$ is $\mathrm{OFF})$.

behavior of the two-CLL NFRP element antenna is obtained as predicted by activation/deactivation of only one of the switches. The maximum simulated and measured values of the radiation efficiency are $80 \%$ in comparison to $70 \%$ for both States 1 and 2, respectively. The maximum value of the measured gain is $4.1 \mathrm{~dB}$ for State 1 (Figure $17(\mathrm{a})$ ) and $3.2 \mathrm{~dB}$ for State 2 (Figure 17(b)).

\section{Conclusion}

In this paper, a family of metamaterial-inspired antennas having reconfigurable radiation patterns was reported. The structures consist of a driven monopole antenna combined with one- and two-capacitively loaded loop (CLL) near field resonant parasitic elements. Two configurations were 


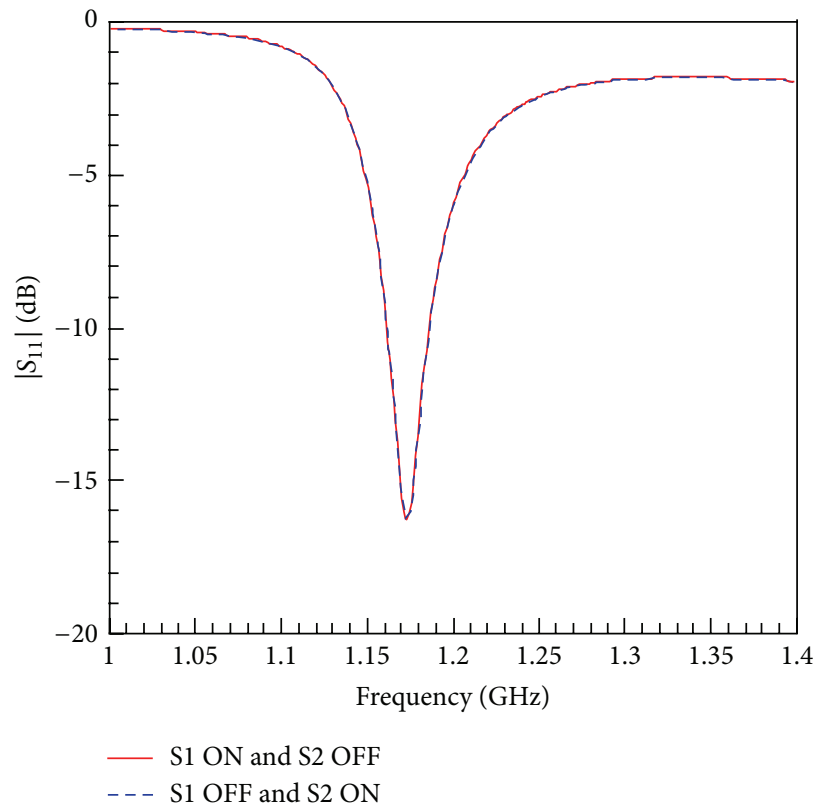

FIGURE 11: Simulated impedance mismatch of the fabricated prototype of the two-CLL NFRP element reconfigurable antenna.

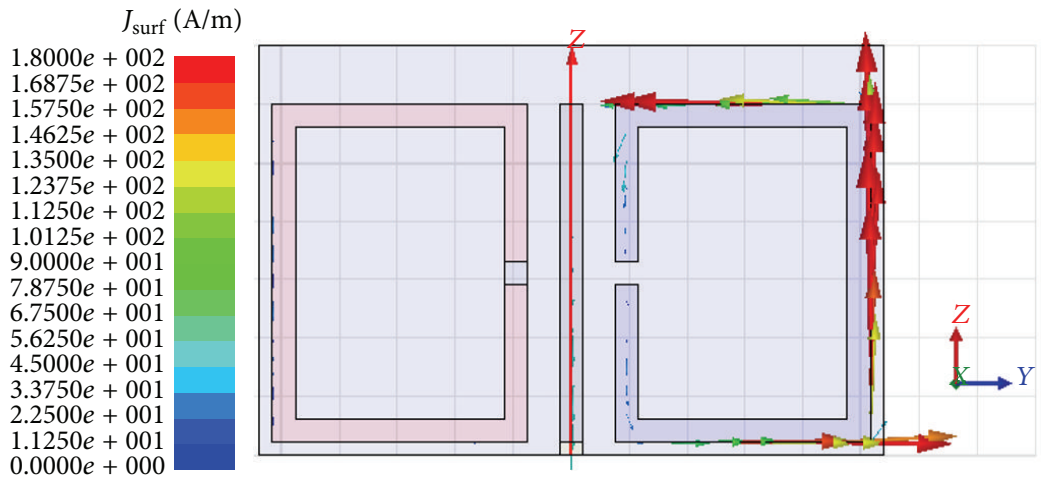

(a)

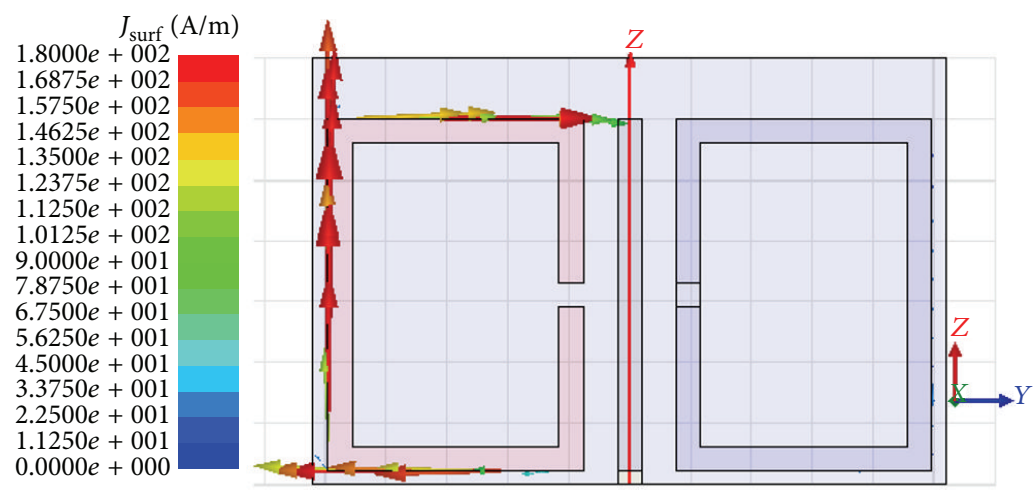

(b)

FIGURE 12: Simulated surface current for the two-element reconfigurable antenna at $f_{R}=1.172 \mathrm{GHz}$ : (a) S1 ON and S2 OFF, and (b) S1 OFF and $\mathrm{S} 2 \mathrm{ON}$. 


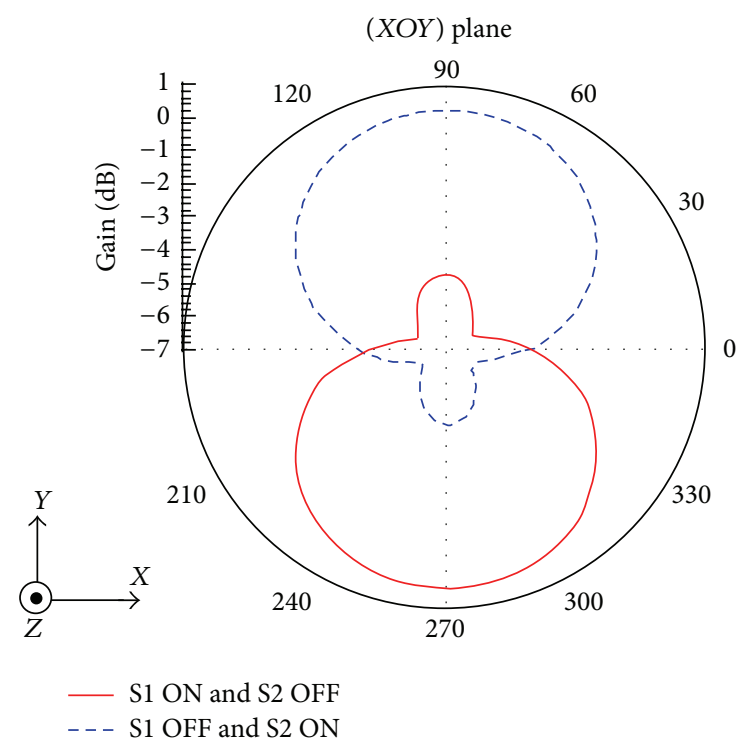

FIGURE 13: Simulated 2D radiation patterns in the (XOY) plane of the prototype two-CLL NFRP element reconfigurable antenna at the operating frequency $f_{R}=1.172 \mathrm{GHz}$.

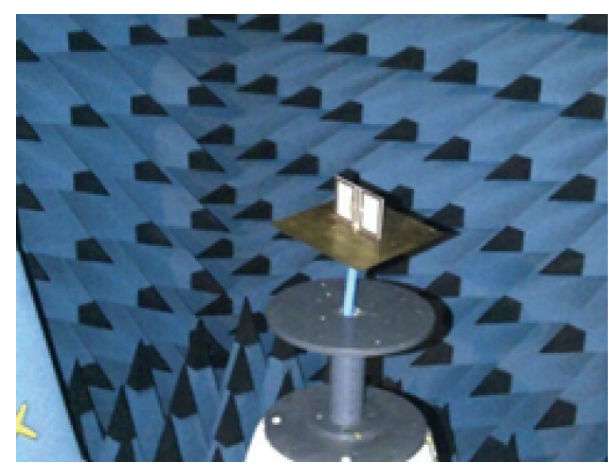

FIGURE 14: Photo of the realized prototype of the two-CLL element reconfigurable antenna.

reported by considering the state of the gaps in the CLL elements, whether they were open or closed. Simulation results show that the structure can change its beam direction by controlling the switched states. Two prototypes with oneand two-CLL NFRP elements were fabricated and tested. The measured results in terms of impedance mismatch and radiation pattern results were presented and discussed. They were found to be in very good agreement with their simulated values. Since the simulations that were presented assumed ideal switches, the results for the radiation efficiencies and realized gains have to be considered as upper bounds. The actual implementation of the switches and the corresponding simulation models would depend on the commercially available technology selected, for example, pin-diodes or MEMs switches. The former could be incorporated with little impact of the overall antenna performance characteristics of the reported prototypes but would require modification of the actual designs and corresponding numerical models

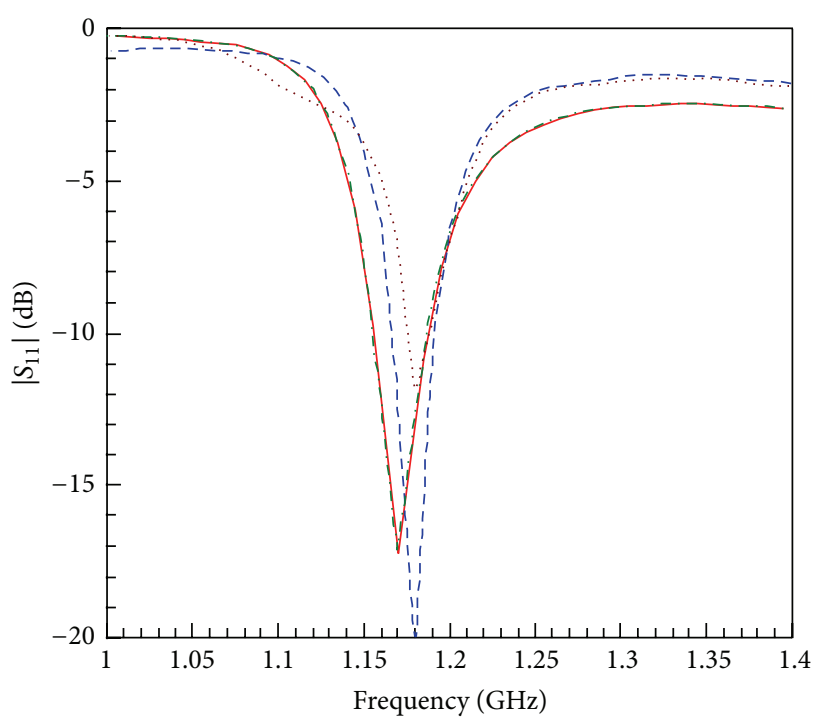

— S1 ON and S2 OFF (simulation)
-- S1 ON and S2 OFF (measurement)
$\ldots$. S1 OFF and S2 ON (simulation)
$\ldots .$. S1 OFF and S2 ON (measurement)

FIGURE 15: Measured and simulated impedance mismatch of the fabricated prototype of the two-CLL NFRP element reconfigurable antenna. State 1 (red) and State 2 (blue).

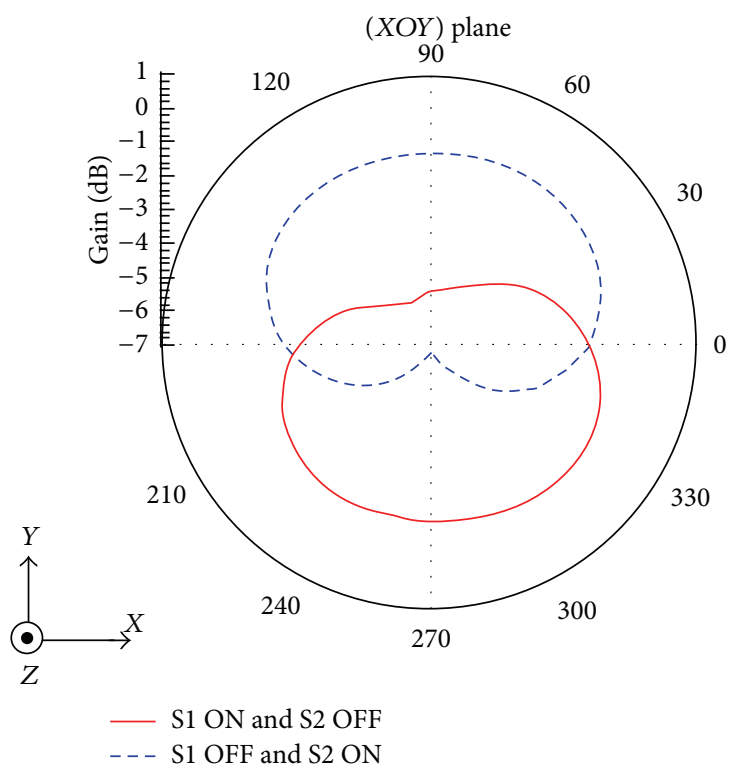

FIGURE 16: Measured 2D radiation patterns of the prototype two NFRP elements reconfigurable at $f_{R}=1.180 \mathrm{GHz}$ : State 1 and State 2.

to account for their associated lumped element capacitance, inductance, and resistance values.

\section{Conflict of Interests}

The authors declare that there is no conflict of interests regarding the publication of this paper. 


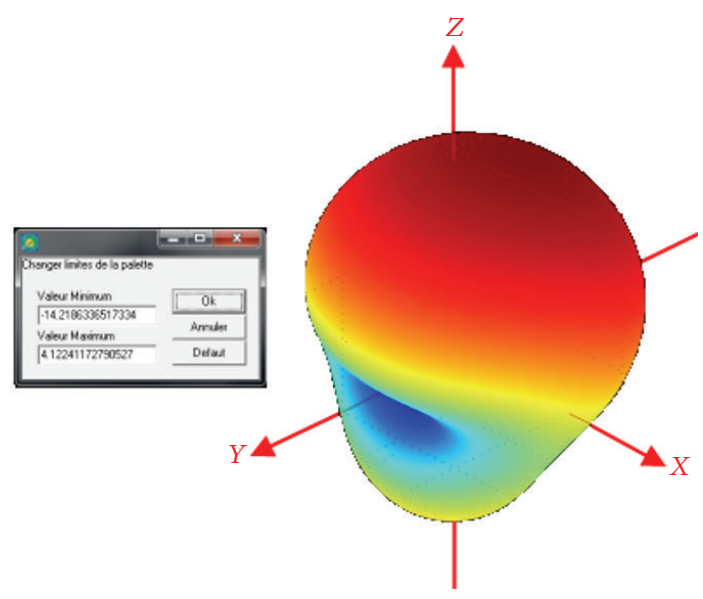

(a)

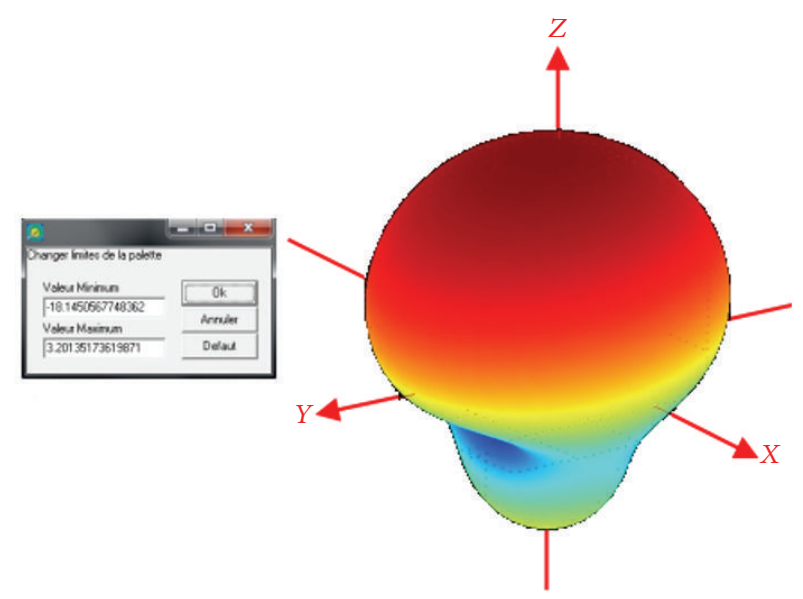

(b)

FIGURE 17: Measured 3D radiation patterns of the prototype two-NFRP element reconfigurable antenna at $f_{R}=1.180 \mathrm{GHz}$ : (a) State 1 and (b) State 2.

\section{Acknowledgment}

This project was funded by the Deanship of Scientific Research (DSR), King Abdulaziz University, under Grant no. 21-135-35-HiCi. The authors, therefore, acknowledge technical and financial support of KAU.

\section{References}

[1] A. Erentok and R. W. Ziolkowski, "Metamaterial-inspired efficient electrically-small antennas," IEEE Transactions on Antennas and Propagation, vol. 56, no. 3, pp. 691-707, 2008.

[2] R. W. Ziolkowski, P. Jin, and C.-C. Lin, "Metamaterial-inspired engineering of antennas," Proceedings of the IEEE, vol. 99, no. 10, pp. 1720-1731, 2011.

[3] N. Engheta and R. W. Ziolkowski, Eds., Metamaterials: Physics and Engineering Explorations, Wiley-IEEE Press, 2006.

[4] Y. Dong and T. Itoh, "Metamaterial-based antennas," Proceedings of the IEEE, vol. 100, no. 7, pp. 2271-2285, 2012.

[5] P. Jin and R. W. Ziolkowski, "Multi-frequency, linear and circular polarized, metamaterial-inspired, near-field resonant parasitic antennas," IEEE Transactions on Antennas and Propagation, vol. 59, no. 5, pp. 1446-1459, 2011.

[6] K. B. Alici and E. Ozbay, "Electrically small split ring resonator antennas," Journal of Applied Physics, vol. 101, no. 8, Article ID 083104, 2007.

[7] K. B. Alici, A. E. Serebryannikov, and E. Ozbay, "Radiation properties and coupling analysis of a metamaterial based, dual polarization, dual band, multiple split ring resonator antenna," Journal of Electromagnetic Waves and Applications, vol. 24, no. 8-9, pp. 1183-1193, 2010.

[8] O. S. Kim and O. Breinbjerg, "Miniaturised self-resonant splitring resonator antenna," Electronics Letters, vol. 45, no. 4, pp. 196-197, 2009.

[9] O. S. Kim, "Low-Q electrically small spherical magnetic dipole antennas," IEEE Transactions on Antennas and Propagation, vol. 58, no. 7, pp. 2210-2217, 2010.
[10] I. K. Kim and V. V. Varadan, "Electrically small, millimeter wave dual band meta-resonator antennas," IEEE Transactions on Antennas and Propagation, vol. 58, no. 11, pp. 3458-3463, 2010.

[11] A. Erentok, P. Luljak, and R. W. Ziolkowski, "Antenna performance near a volumetric metamaterial realization of an artificial magnetic conductor," IEEE Transactions on Antennas and Propagation, vol. 53, pp. 160-172, 2005.

[12] Y. Zhang, W. Hong, C. Yu, Z.-Q. Kuai, Y.-D. Don, and J.-Y. Zhou, "Planar ultrawideband antennas with multiple notched bands based on etched slots on the patch and/or split ring resonators on the feed line," IEEE Transactions on Antennas and Propagation, vol. 56, no. 9, pp. 3063-3068, 2008.

[13] M.-C. Tang, S. Xiao, T. Deng et al., "Compact UWB antenna with multiple band-notches for WiMAX and WLAN," IEEE Transactions on Antennas and Propagation, vol. 59, no. 4, pp. 1372-1376, 2011.

[14] C.-C. Lin, P. Jin, and R. W. Ziolkowski, "Single, dual and triband-notched ultrawideband (UWB) antennas using capacitively loaded loop (CLL) resonators," IEEE Transactions on Antennas and Propagation, vol. 60, no. 1, pp. 102-109, 2012.

[15] J. T. Bernhard, Reconfigurable Antennas, Morgan \& Claypool Publishers, San Rafael, Calif, USA, 2007.

[16] R. L. Haupt and M. Lanagan, "Reconfigurable antennas," IEEE Antennas and Propagation Magazine, vol. 55, no. 1, pp. 49-61, 2013.

[17] A. E. Fathy, A. Rosen, H. S. Owen et al., "Silicon-based reconfigurable antennas-concepts, analysis, implementation, and feasibility," IEEE Transactions on Microwave Theory and Techniques, vol. 51, no. 6, pp. 1650-1660, 2003.

[18] G. Wang, T. Polley, A. Hunt, and J. Papapolymerou, "A high performance tunable RF MEMS switch using barium strontium titanate (BST) dielectrics for reconfigurable antennas and phased arrays," IEEE Antennas and Wireless Propagation Letters, vol. 4, no. 1, pp. 217-220, 2005.

[19] N. Behdad and K. Sarabandi, "A varactor-tuned dual-band slot antenna," IEEE Transactions on Antennas and Propagation, vol. 54, no. 2, pp. 401-408, 2006.

[20] Y. Tawk, J. Costantine, S. Hemmady, G. Balakrishnan, K. Avery, and C. G. Christodoulou, "Demonstration of a cognitive radio 
front end using an optically pumped reconfigurable antenna system (OPRAS)," IEEE Transactions on Antennas and Propagation, vol. 60, no. 2, pp. 1075-1083, 2012.

[21] S. Dakhli, J.-M. Floch, K. Mahdjoubi, H. Rmili, and H. Zangar, "Compact and multi-band metamaterial-inspired dipole antenna," in Proceedings of the 7th European Conference on Antennas and Propagation (EuCAP '13), pp. 2765-2768, Gothenburg, Sweden, April 2013.

[22] O. Turkmen, G. Turhan-Sayan, and R. W. Ziolkowski, "Metamaterial inspired, electrically small, GSM antenna with steerable radiation patterns and high radiation efficiency," in Proceedings of the IEEE Antennas and Propagation Society International Symposium (APSURSI '13), pp. 770-771, July 2013.

[23] M. Barbuto, F. Bilotti, and A. Toscano, "Design of a multifunctional SRR-loaded printed monopole antenna," International Journal of RF and Microwave Computer-Aided Engineering, vol. 22, no. 4, pp. 552-557, 2012. 

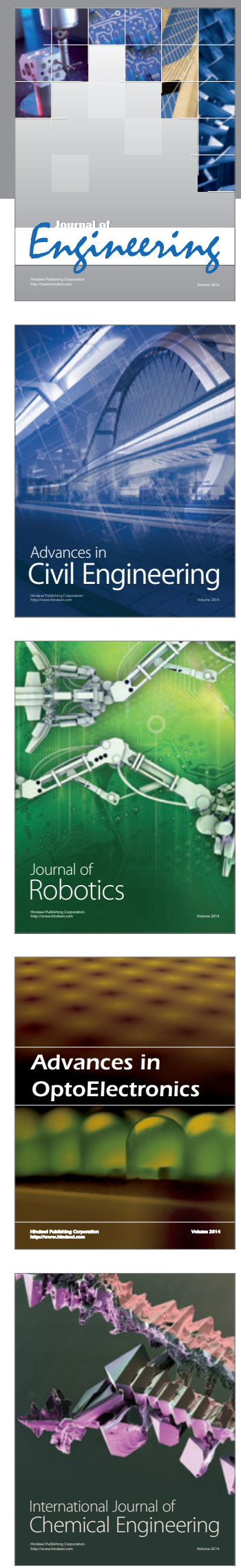

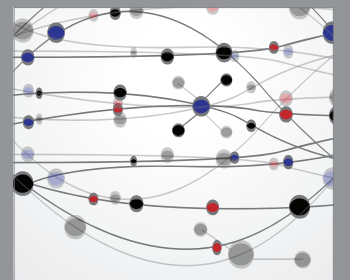

The Scientific World Journal
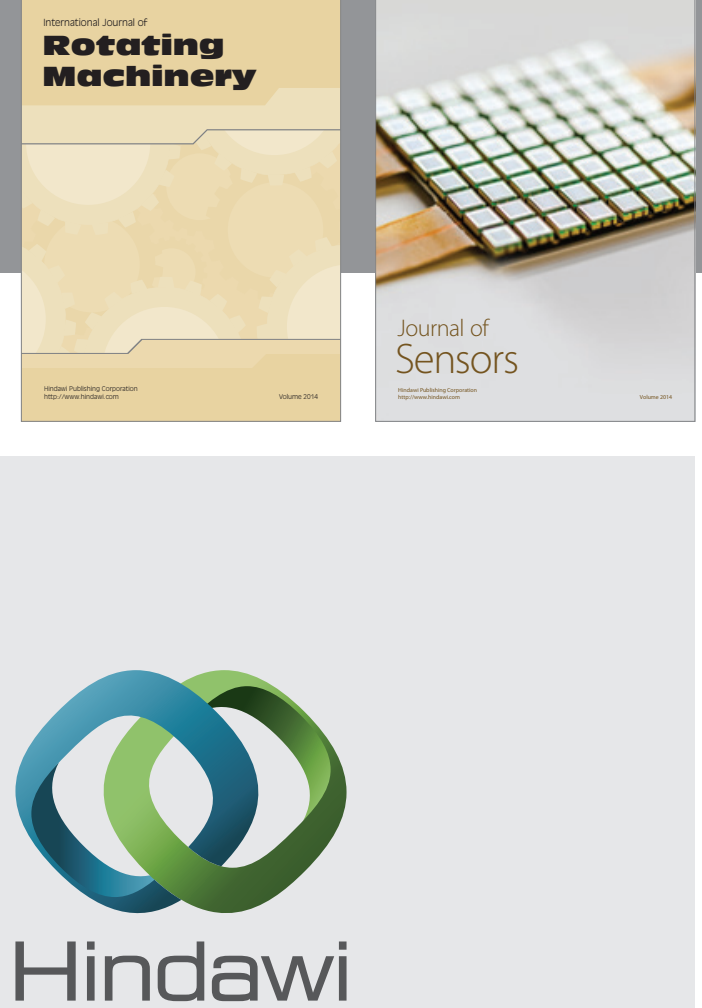

Submit your manuscripts at http://www.hindawi.com
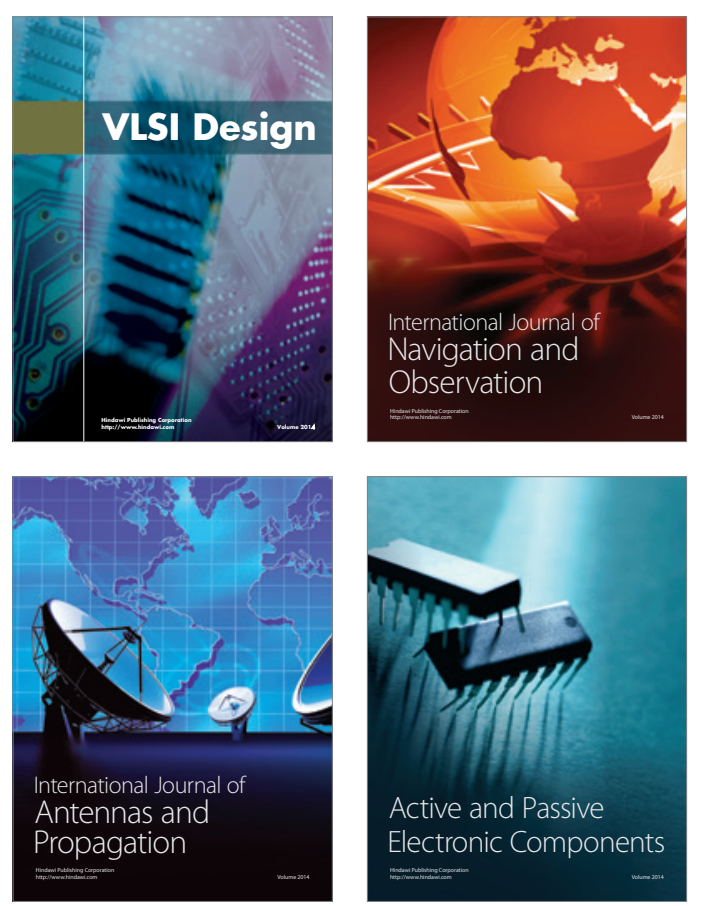
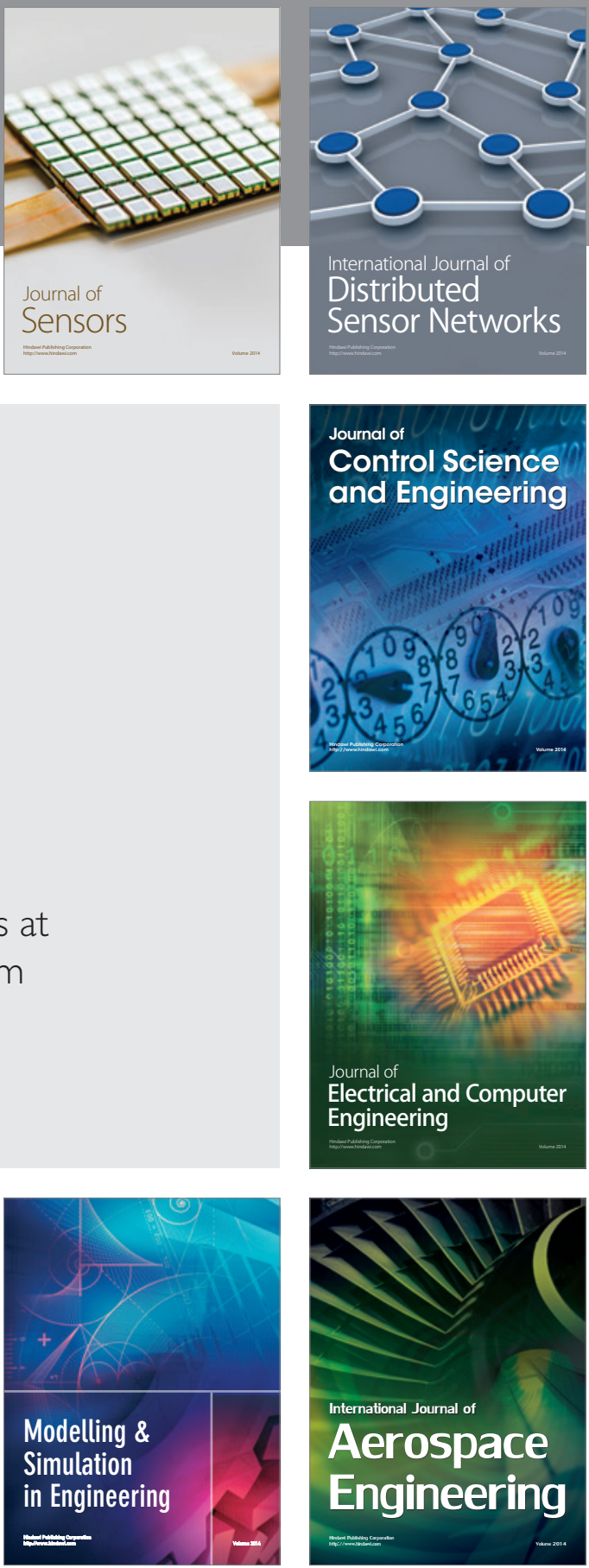

Journal of

Control Science

and Engineering
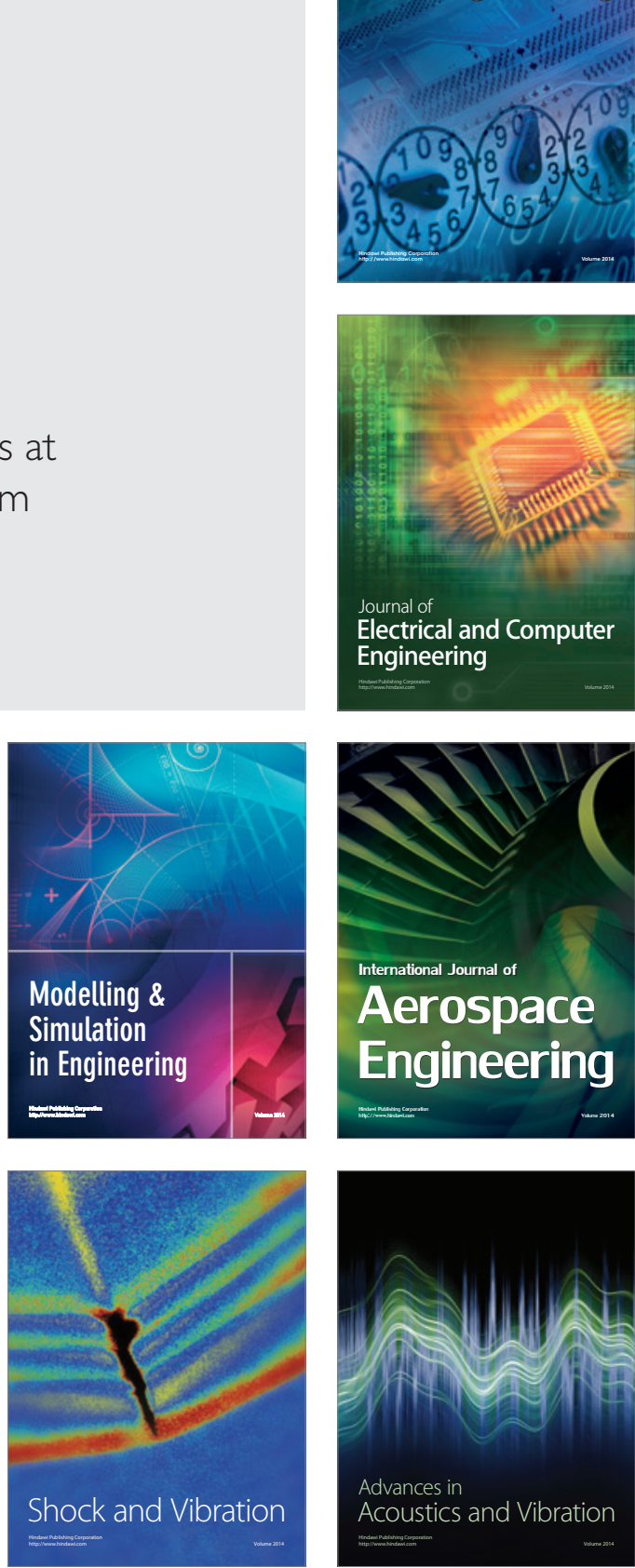